\title{
SIGNED SUMS OF TERMS OF A SEQUENCE
}

\author{
FENG-JUAN CHEN AND YONG-GAO CHEN
}

(Communicated by Matthew A. Papanikolas)

\begin{abstract}
We give a sufficient and necessary condition on the sequence $\left\{a_{n}\right\}$ of integers that for any integer $l \geq 1$, every integer can be represented in the form $\varepsilon_{l} a_{l}+\varepsilon_{l+1} a_{l+1}+\cdots+\varepsilon_{k} a_{k}$, where $\varepsilon_{i} \in\{-1,1\}(i=l, l+1, \ldots, k)$. This generalizes the known result on integral-valued polynomial values. Moreover, we show that such sequences exist with any growth rate. This answers two problems posed by Bleicher. We also pose several problems for further research.
\end{abstract}

\section{INTRODUCTION}

In [1], Bleicher proved the following interesting result: for any given integer $k \geq 2$, every integer can be represented in the form

$$
n=\varepsilon_{1} 1^{k}+\varepsilon_{2} 2^{k}+\cdots+\varepsilon_{t} t^{k}, \quad \varepsilon_{i} \in\{-1,1\}, \quad i=1,2, \ldots, t .
$$

$\mathrm{Yu} 11$ generalized the result to integral-valued polynomials with fixed divisor equal to 1. Boulanger and Chabert [2] considered the problem in the ring of algebraic integers $O_{K}$ of a cyclotomic field $K$. For related research, one may refer to [3, 44, [5], 7], 8] and [9]. Starting from the result on $k$ th powers, Bleicher [1] posed the following two problems.

Problem 1. Does there exist a constant $c>1$ and an increasing sequence of integers $\left\{a_{i}\right\}$ with $a_{i}>c^{i}$ for every positive integer $i$ such that for every positive integer $n$, there is a positive integer $m$ and a choice of $\varepsilon_{i}= \pm 1$ for which $n=$ $\sum_{i=1}^{m} \varepsilon_{i} a_{i}$ ?

Problem 2. On the assumption that Problem 1 is answered affirmatively, is there an upper bound for possible choices of $c$ ?

For sequences, Erdős and Surányi [6] proved the following result: Let $0<a_{1}<$ $a_{2}<\cdots$ be a sequence of integers. If (a) the sequence contains infinitely many odd numbers; (b) for some $m$, all positive integers $>m$ can be represented as the sum of different elements of the sequence; (c) $a_{n+1}<2 a_{n}-m$ for $n \geq n_{0}$, then every integer can be represented in the form $\varepsilon_{1} a_{1}+\varepsilon_{2} a_{2}+\cdots+\varepsilon_{t} a_{t}$, where $\varepsilon_{i} \in\{-1,1\}(i=1,2, \ldots, t)$.

In this note we prove the following result. This generalizes the result on integralvalued polynomials (Bleicher [1], Yu [1]). Moreover, we answer Problem 1 affirmatively and Problem 2 negatively.

Received by the editors March 14, 2011 and, in revised form, August 4, 2011.

2010 Mathematics Subject Classification. Primary 11A67, 11B50, 11B83, 11P05.

Key words and phrases. Signed sums, sequences, polynomials.

This work was supported by the National Natural Science Foundation of China, Grant No. 11071121 and the Project of Graduate Education Innovation of Jiangsu Province (CXZZ110868).

(c) 2012 American Mathematical Society Reverts to public domain 28 years from publication 
Theorem 1. Let $a_{1}, a_{2}, \ldots$ be a sequence of integers. Then for any integers $l \geq 1$ and $n$, there exists an integer $t \geq l$ and a choice of $\varepsilon_{i}= \pm 1$ such that

$$
n=\varepsilon_{l} a_{l}+\varepsilon_{l+1} a_{l+1}+\cdots+\varepsilon_{t} a_{t}
$$

if and only if the following two conditions hold:

(i) there exists a nonzero integer $M$ such that for any $l \geq 1$ there exists an integer $r=r_{l} \geq l$ and $\varepsilon_{i} \in\{-1,1\}(l \leq i \leq r)$ with $M=\varepsilon_{l} a_{l}+\cdots+\varepsilon_{r} a_{r}$;

(ii) for any $l \geq 1$ we have $\operatorname{gcd}\left(a_{l}, a_{l+1}, \ldots\right)=1$.

Remark. For any $l \geq 1$, letting $r=r_{l}$ and $s=r_{r+1}$, we have

$$
0=M-M=\varepsilon_{l} a_{l}+\cdots+\varepsilon_{r} a_{r}-\varepsilon_{r+1} a_{r+1}-\cdots-\varepsilon_{s} a_{s} .
$$

So, for a fixed $l \geq 1$ and a fixed integer $n$, the $t$ in Theorem 1 can take infinitely many values.

Remark. Let $a_{1}=2, a_{2}=3$ and $a_{i+2}=a_{i+1}+a_{i}+1(i=1,2, \ldots)$. Since $1=$ $-a_{i}-a_{i+1}+a_{i+2}$, (i) is true for $M=1$ and (ii) is true by $\left(a_{i}, a_{i+1}, a_{i+2}\right)=1$ for all $i \geq 1$. This verifies Theorem 11. One may easily prove that $a_{i}>(\sqrt{2})^{i}$ for all $i \geq 1$. Thus Problem 1 is answered affirmatively.

Remark. For an integral-valued polynomial $f(x)$ of degree $h$ with fixed divisor equal to 1 , let $a_{i}=f(i)(i=1,2, \ldots)$. For any integers $l$ and $n$, by the Lagrange interpolation formula we know that $f(n)$ is a combination of $f(l), f(l+1), \ldots$, $f(l+h)$ with integral coefficients. Since $f(x)$ has no fixed factors, we have

$$
\operatorname{gcd}(f(l), f(l+1), \ldots, f(l+h))=1 .
$$

On the other hand, let $f_{1}(x)=f(x+1)-f(x), f_{i+1}(x)=f_{i}\left(x+2^{i}\right)-f_{i}(x)(i=$ $1,2, \ldots)$. Then $f_{i}(x)$ is a polynomial of degree $h-i(1 \leq i \leq h)$. So $f_{h}(x)$ is a nonzero constant. We also have

$$
f_{h}(x)=\sum_{i=0}^{2^{h}-1} \varepsilon_{i} f(x+i), \quad \varepsilon_{i} \in\{-1,1\} .
$$

Since $f(x)$ is integral-valued, $f_{h}(x)=f_{h}(0)$ is an integer. For related information, one may refer to [10]. By Theorem 11 for any $l \geq 1$, there exists $t \geq l$ such that every integer can be represented in the form $n=\varepsilon_{l} f(l)+\varepsilon_{l+1} f(l+1)+\cdots+\varepsilon_{t} f(t)$, where $\varepsilon_{i} \in\{-1,1\}(i=l, l+1, \ldots, t)$. That is the main result in [11].

For Problems 1 and 2, we have the following general result. This means that sequences exist with any growth rate.

Theorem 2. For any sequence $1<c_{1}<c_{2}<\cdots$, there exists a sequence $1<$ $a_{1}<a_{2}<\cdots$ of integers with $a_{i}>c_{i}$ for every positive integer $i$ such that for any integers $l \geq 1$ and $n$, there are infinitely many positive integers $m \geq l$ for which there is a choice of $\varepsilon_{i}= \pm 1$ with $n=\sum_{i=l}^{m} \varepsilon_{i} a_{i}$.

Remark. For any given $C>1$, let $c_{i}=C^{i}$. Problems 1 and 2 immediately follow from Theorem 2

In the proof of Theorem 2, some consecutive terms are "near". If we require that consecutive terms are not "near", i.e. $a_{k+1} \geq \alpha a_{k}$ for all $k \geq 1$, what is the largest possible value of $\alpha$ ? We have the following precise result. 
Theorem 3. (i) If $\left\{a_{i}\right\}$ is a sequence of positive integers such that, for some integer $n_{0}$ and infinitely many positive integers $l$, there is an integer $m>l$ and a choice of $\varepsilon_{i}= \pm 1$ with

$$
\sum_{i=l}^{m} \varepsilon_{i} a_{i}=n_{0},
$$

then there are infinitely many positive integers $k$ such that $a_{k+1} \leq 2 a_{k}-1$.

(ii) Let $a_{1}$ be any positive integer, and define the sequence $\left\{a_{i}\right\}$ by $a_{k+1}=2 a_{k}-1$ for all $k \geq 1$. Then for any integers $l \geq 1$ and $n$, there are infinitely many positive integers $m>l$ for which there is a choice of $\varepsilon_{i}= \pm 1$ with

$$
n=\sum_{i=l}^{m} \varepsilon_{i} a_{i} .
$$

Now we consider only representations of the form

$$
n=\sum_{i=1}^{m} \varepsilon_{i} a_{i} .
$$

For this purpose we introduce the following definition.

Definition 1. Let $M$ be a positive integer. The subsequence $\left\{a_{l}, a_{l+1}, \ldots, a_{k}\right\}(k \geq$ $l)$ is said to be $M$-coprime if $\operatorname{gcd}\left(M, a_{l}, a_{l+1}, \ldots, a_{k}\right)=1$.

The condition (ii) in Theorem 1 implies that there are infinitely many disjoint $M$-coprime subsequences.

Theorem 4. Let $a_{1}, a_{2}, \ldots$ be a sequence of integers satisfying

(i) there exists a positive integer $M$ such that for any $l \geq 1$ there exists an integer $r=r_{l} \geq l$ and $\varepsilon_{i} \in\{-1,1\}(l \leq i \leq r)$ with

$$
M=\varepsilon_{l} a_{l}+\cdots+\varepsilon_{r} a_{r}
$$

(ii) there are at least $M$ disjoint $M$-coprime subsequences of $\left\{a_{1}, a_{2}, \ldots\right\}$.

Then every integer can be represented in the form

$$
n=\varepsilon_{1} a_{1}+\varepsilon_{2} a_{2}+\cdots+\varepsilon_{t} a_{t}, \quad \varepsilon_{i} \in\{-1,1\}, \quad i=1,2, \ldots, t .
$$

\section{Proof of theOrems}

Proof of Theorem 1. First we assume that for any integers $l \geq 1$ and $n$, there exists an integer $t \geq l$ and a choice of $\varepsilon_{i}= \pm 1$ such that

$$
n=\varepsilon_{l} a_{l}+\varepsilon_{l+1} a_{l+1}+\cdots+\varepsilon_{t} a_{t} .
$$

We take $n=1$. Then (i) holds with $M=1$. (ii) follows from

$$
\operatorname{gcd}\left(a_{l}, a_{l+1}, \ldots, a_{t}\right)=1 .
$$

Now we assume that both (i) and (ii) hold.

For any $l \geq 1$, let $r=r_{l}$ and $s=r_{r+1}$. We have

$$
2 M=\varepsilon_{l} a_{l}+\cdots+\varepsilon_{r} a_{r}+\varepsilon_{r+1} a_{r+1}+\cdots+\varepsilon_{s} a_{s} .
$$

So we may assume that $M$ is a positive even number. 
Fix an integer $l \geq 1$. Let

$$
A_{k}=\left\{\varepsilon_{l} a_{l}+\varepsilon_{l+1} a_{l+1}+\cdots+\varepsilon_{k} a_{k}: \varepsilon_{i} \in\{-1,1\}(l \leq i \leq k)\right\}, \quad k=l, l+1, \ldots
$$

and

$$
\bar{A}_{k}=\left\{b: 0 \leq b \leq M-1, b \equiv a(\bmod M), a \in A_{k}\right\}, \quad k=l, l+1, \ldots
$$

Let $m_{k}=\left|\bar{A}_{k}\right|$. Since the numbers in $A_{k}$ have the same parity, we know that the numbers in $\bar{A}_{k}$ have the same parity. Thus $1 \leq m_{k} \leq M / 2$ for all $k \geq l$. It is clear that $m_{l} \leq m_{l+1} \leq \cdots$. So there exist two positive integers $m$ and $i_{0}>l$ such that $m_{i}=m$ for all $i \geq i_{0}$. Let

$$
\bar{A}_{i}=\left\{b_{i 1}, b_{i 2}, \ldots, b_{i m}\right\}, \quad i \geq i_{0} .
$$

By $\left|\bar{A}_{i+1}\right|=m$, we have

$$
\begin{aligned}
& \left(b_{i 1}-a_{i+1}\right)+\left(b_{i 2}-a_{i+1}\right)+\cdots+\left(b_{i m}-a_{i+1}\right) \\
& \quad \equiv\left(b_{i 1}+a_{i+1}\right)+\left(b_{i 2}+a_{i+1}\right)+\cdots+\left(b_{i m}+a_{i+1}\right)(\bmod M) .
\end{aligned}
$$

Thus $2 m a_{i+1} \equiv 0(\bmod M)$ for all $i \geq i_{0}$. By (ii) we have $2 m \equiv 0(\bmod M)$. Since $1 \leq m \leq M / 2$, we have $m=M / 2$. Again, by (ii), there exists an integer $j \geq i_{0}$ such that $a_{j+1}$ is odd. Hence

$$
\bar{A}_{j} \cup \bar{A}_{j+1}=\{0,1, \ldots, M-1\} .
$$

Thus, for any integer $n$, there exists $k=j$ or $j+1, \varepsilon_{i} \in\{-1,1\}(i=l, l+1, \ldots, k)$ and an integer $u$ such that

$$
n=\varepsilon_{l} a_{l}+\varepsilon_{l+1} a_{l+1}+\cdots+\varepsilon_{k} a_{k}+u M .
$$

By (i) we obtain a proof of Theorem 1 .

Proof of Theorem 2. Let

$$
a_{2 i-1}=\left[c_{2 i}\right]+2 i+1, \quad a_{2 i}=\left[c_{2 i}\right]+2 i+2, \quad i=1,2, \ldots .
$$

Then $a_{2 i+1}>a_{2 i}>a_{2 i-1}>\left[c_{2 i}\right]+1>c_{2 i}>c_{2 i-1}$ for all $i \geq 1$.

Let $l, n$ be two integers with $l \geq 1$. For any integer $j>l+|n|$ we have $n+a_{l}+$ $\cdots+a_{2 j}>n+2 j>0$. Let $t=n+\sum_{i=l}^{2 j} a_{i}$. Then

$$
n=\sum_{i=l}^{2 j}\left(-a_{i}\right)+\sum_{i=j+1}^{j+t}\left(-a_{2 i-1}+a_{2 i}\right) .
$$

Hence, for every integer $n$ there are infinitely many positive integers $m>l$ for which there is a choice of $\varepsilon_{i}= \pm 1$ with $n=\sum_{i=l}^{m} \varepsilon_{i} a_{i}$. This completes the proof of Theorem 2 ,

Proof of Theorem [3. (i) Suppose that $\left\{a_{n}\right\}$ is a sequence of positive integers satisfying the condition, but $a_{k+1} \geq 2 a_{k}$ for all $k \geq k_{0}$. Then $a_{k} \rightarrow+\infty$ as $k \rightarrow+\infty$. Take an integer $l \geq k_{0}$ with $a_{l}>\left|n_{0}\right|$ for which there is an integer $m>l$ and a choice of $\varepsilon_{i}= \pm 1$ with

$$
\sum_{i=l}^{m} \varepsilon_{i} a_{i}=n_{0}
$$


Thus

$$
\begin{aligned}
a_{m} & =\varepsilon_{m} n_{0}+\sum_{i=l}^{m-1}-\varepsilon_{m} \varepsilon_{i} a_{i} \leq\left|n_{0}\right|+\sum_{i=l}^{m-1} a_{i} \\
& <a_{l}+\sum_{i=l}^{m-1} a_{i}=2 a_{l}+\sum_{i=l+1}^{m-1} a_{i} \\
& \leq a_{l+1}+\sum_{i=l+1}^{m-1} a_{i}=2 a_{l+1}+\sum_{i=l+2}^{m-1} a_{i} \\
& \leq \cdots \\
& \leq 2 a_{m-2}+a_{m-1} \leq 2 a_{m-1},
\end{aligned}
$$

a contradiction with $a_{k+1} \geq 2 a_{k}$ for all $k \geq k_{0}$. This completes the proof of Theorem 3 (i).

(ii) Let $l \geq 1$ and $n$ be two integers. For any integer $t>l+|n|$, let $s=$ $n+a_{l}+a_{l+1}+\cdots+a_{t}$. Then by $a_{i} \geq 1$ for all $i \geq 1$ we have $s \geq n+t-l+1>0$. By $a_{k+1}=2 a_{k}-1$ we have

$$
\begin{aligned}
a_{t+s} & =a_{t+s-1}+a_{t+s-1}-1=a_{t+s-1}+a_{t+s-2}+a_{t+s-2}-2 \\
& =\cdots \\
& =a_{t+s-1}+a_{t+s-2}+\cdots+a_{t}+a_{t}-s \\
& =a_{t+s-1}+a_{t+s-2}+\cdots+a_{t}-a_{t-1}-a_{t-2}-\cdots-a_{l}-n .
\end{aligned}
$$

Thus

$$
n=-\sum_{i=l}^{t-1} a_{i}+\sum_{i=t}^{t+s-1} a_{i}-a_{t+s} .
$$

This completes the proof of Theorem 3 (ii).

Proof of Theorem 4. If $M=1$, then Theorem 4 is clear. Now we assume that $M>1$. Let the notation be as in the proof of Theorem 1 (the fixed $l$ is equal to 1 and we do not assume that $M$ is even). Let $i_{0}=0$ and let $\left\{a_{i_{j-1}+1}, \ldots, a_{i_{j}}\right\}(j=$ $1,2, \ldots, M)$ be $M$ disjoint $M$-coprime subsequences of the sequence $\left\{a_{i}\right\}$. It is clear that $m_{i_{1}} \geq 1$. If $m_{i_{2}}=m_{i_{1}}$, then, similarly to the proof of Theorem 1, we have

$$
2 m_{i_{1}} a_{j} \equiv 0(\bmod M), \quad j=i_{1}+1, \ldots, i_{2} .
$$

Since $\left\{a_{i_{1}+1}, \ldots, a_{i_{2}}\right\}$ is an $M$-coprime subsequence, we have $2 m_{i_{1}} \equiv 0(\bmod M)$. If $M$ is odd, then $m_{i_{1}}=M$. If $M$ is even, then, similarly to the proof of Theorem 1, we have $m_{i_{1}}=M / 2$. Hence, if $m_{i_{1}} \neq M, M / 2$, then $m_{i_{2}}>m_{i_{1}}$. Continuing these arguments, we have that if $M$ is odd, then $m_{i_{M}}=M$. Thus $\bar{A}_{i_{M}}=\{0,1, \ldots, M-1\}$. If $M$ is even, then $m_{i_{M / 2}}=M / 2$ and $m_{i} \leq M / 2$ for all $i$. Since $\left\{a_{i_{M / 2}+1}, \ldots, a_{i_{(M / 2)+1}}\right\}$ is an $M$-coprime subsequence, there exists $j_{0} \in$ $\left\{i_{M / 2}+1, \ldots, i_{(M / 2)+1}\right\}$ such that $a_{j_{0}}$ is odd. Since $m_{i_{M / 2}} \leq m_{j_{0}-1} \leq m_{j_{0}} \leq M / 2$, we have $m_{j_{0}-1}=m_{j_{0}}=M / 2$. Since the parities of the numbers in $\bar{A}_{j_{0}-1}$ and in $\bar{A}_{j_{0}}$ are distinct, we have

$$
\bar{A}_{j_{0}-1} \cup \bar{A}_{j_{0}}=\{0,1, \ldots, M-1\} .
$$

Now the following arguments are similar to those of Theorem 11. This completes the proof of Theorem 4 . 


\section{Final REMARKS}

For a sequence $A=\left\{a_{i}\right\}$ (finite or infinite) and an integer $n$, let $\sigma_{A}(n)$ be the ways of representation of $n=\varepsilon_{1} a_{1}+\varepsilon_{2} a_{2}+\cdots+\varepsilon_{t} a_{t}, \quad \varepsilon_{i} \in\{-1,1\}, \quad i=1,2, \ldots, t$. In the previous arguments all $A$ satisfy $\sigma_{A}(n)=+\infty$. It is natural to ask: is there a sequence $A=\left\{a_{i}\right\}$ such that $1 \leq \sigma_{A}(n)<+\infty$ for all integers $n$ ? Now we construct such a sequence.

For $A_{k}=\left\{a_{1}, a_{2}, \ldots, a_{k}\right\}$, let

$$
\Delta\left(A_{k}\right)=\left\{\varepsilon_{1} a_{1}+\varepsilon_{2} a_{2}+\cdots+\varepsilon_{k} a_{k}: \varepsilon_{i} \in\{-1,1\}, \quad i=1,2, \ldots, k\right\} .
$$

Let $a_{1}=1, a_{2}=3, a_{3}=4$. Then $\Delta\left(A_{1}\right)=\{ \pm 1\}, \Delta\left(A_{2}\right)=\{ \pm 2, \pm 4\}$ and $\Delta\left(A_{3}\right)=\{0, \pm 2, \pm 6, \pm 8\}$. Suppose that we have $a_{1}, \ldots, a_{k}(k \geq 3)$. Let $n_{k}$ be the least positive integer which is not in $\Delta\left(A_{1}\right) \cup \cdots \cup \Delta\left(A_{k}\right)$, and let $a_{k+1}=a_{1}+$ $\cdots+a_{k}+n_{k}$. Then $n_{k} \in \Delta\left(A_{k+1}\right)$ and each positive integer in $\Delta\left(A_{k+1}\right)$ is at least $a_{k+1}-\left(a_{1}+\cdots a_{k}\right)=n_{k}$. Thus we obtain two sequences, $A=\left\{a_{i}\right\}_{i=1}^{\infty}$ and $\left\{n_{i}\right\}_{i=3}^{\infty}$, such that $3=n_{3}<n_{4}<\cdots$ and for every $k \geq 3$ we have $\sigma_{A}(n)=\sigma_{A_{k}}(n) \geq 1$ for all $n<n_{k}$.

Now we have proved the following theorem.

Theorem 5. There exists a strictly increasing sequence $A=\left\{a_{i}\right\}_{i=1}^{\infty}$ of positive integers such that $1 \leq \sigma_{A}(n)<+\infty$ for all integers $n$.

We pose the following problems here for further research.

Problem 3. Is there any (strictly increasing) sequence $A=\left\{a_{i}\right\}$ of positive integers such that $\sigma_{A}(0)=2$ and $\sigma_{A}(n)=1$ for all integers $n$ ?

Problem 4. Is there a constant $c>1$ and a (strictly increasing) sequence $A=\left\{a_{i}\right\}$ of positive integers such that $1 \leq \sigma_{A}(n) \leq c$ for all integers $n$ ?

If $A=\left\{1,3,3^{2}, \ldots\right\}$, then $\sigma_{A}(n) \in\{0,1\}$ for all integers $n$. We pose the following problem.

Problem 5. Is there any (strictly increasing) sequence $A=\left\{a_{i}\right\}$ of positive integers such that $\sigma_{A}(n) \geq 1$ for all integers $n$ and $\sigma_{A}(n)=1$ for infinitely many integers $n$ ?

Added in proof. We have known that Problem 3 is negative and that Problems 4 and 5 are affirmative.

Added after posting. We find that Problem 4 is still open.

\section{ACKNOWLEDGEMENT}

We are grateful to the referee for detailed comments.

\section{REFERENCES}

[1] M. N. Bleicher, On Prielipp's problem on signed sums of $k$ th powers, J. Number Theory 56 (1996), 36-51. MR 1370195 (96j:11011)

[2] J. Boulanger and J. L. Chabert, On the representation of integers as linear combinations of consecutive values of a polynomial, Trans. Amer. Math. Soc. 356 (2004), 5071-5088. MR2084411(2005d:11154)

[3] J. W. S. Cassels, On the representation of integers as the sums of distinct summands taken from a fixed set, Acta Sci. Math. Szeged 21 (1960), 111-124. MR0130236 (24:A103)

[4] Y. G. Chen, On subset sums of a fixed set, Acta Arith. 106 (2003), no. 3, 207-211. MR.1957105 (2003j:11019) 
[5] P. Erdős and R. L. Graham, Old and new problems and results in combinatorial number theory, Monographie 28 de L'enseignement mathématique, Geneva, 1980. MR592420|(82j:10001)

[6] P. Erdős and J. Surányi, Über ein Problem aus der additiven Zahlentheorie, Mat. Lapok 10 (1959), 284-290. MR0125825 (23:A3122)

[7] R. L. Graham, Complete sequences of polynomial values, Duke Math. J. 31 (1964), 275-285. MR.0162759(29:63)

[8] N. Hegyvári, On the representation of integers as sums of distinct terms from a fixed set, Acta Arith. 92 (2000), 99-104. MR:1750309 (2001c:11014)

[9] T. Łuczak and T. Schoen, On the maximal density of sum-free sets, Acta Arith. 95 (2000), 225-229. MR $1793162(2001 \mathrm{k}: 11018)$

[10] M. B. Nathanson, Elementary Methods in Number Theory, Springer-Verlag, New York, 2000. MR.1732941 (2001j:11001)

[11] H. B. Yu, Signed sums of polynomial values, Proc. Amer. Math. Soc. 130 (2002), 1623-1627. MR:1887008(2002m:11007)

School of Mathematical Sciences and Institute of Mathematics, Nanjing Normal University, Nanjing 210046, People's Republic of China - and - Department of Mathematics, Suzhou University, Suzhou 215006, People's Republic of China

E-mail address: cfjsz@126.com

School of Mathematical Sciences and Institute of Mathematics, Nanjing Normal University, Nanjing 210046, People's Republic of China

E-mail address: ygchen@njnu.edu.cn 